Supporting Information for

\title{
CNTs Coated Conductive Elastomer: Electrical and Near Infrared Light Dual-Stimulated Shape Memory, Self-Healing and Wearable Sensing
}

Fei Wang, Cheng Zhang, and Xuejuan Wan*

Shenzhen Key Laboratory of Polymer Science and Technology, Guangdong Research Center for Interfacial Engineering of Functional Materials, College of Materials Science and Engineering, Shenzhen University, Shenzhen 518060, PR China.

Corresponding Author: wanxj@szu.edu.cn. 
Synthesis of CNTs-Based RAFT Agent (CNTs-CTA): Typically, Bi-Br was first fabricated via the reaction of 2-bromoisobutyryl bromide and APTES on the basis of previous literature. Then $\mathrm{Bi}-\mathrm{Br}(1.0 \mathrm{~g}, 2.7 \mathrm{mmol})$, methyl 3-mercaptopropionate $(0.33$ $\mathrm{g}, 2.7 \mathrm{mmol}), \mathrm{CS}_{2}(0.62 \mathrm{~g}, 0.8 \mathrm{mmol})$ and $\mathrm{Et}_{3} \mathrm{~N}(0.27 \mathrm{~g}, 2.7 \mathrm{mmol})$ were added into acetone $(10.0 \mathrm{~mL})$ with stirring for $12 \mathrm{~h}$ at room temperature. After reaction was suspended, the reaction mixture was handled by rotary evaporation to remove the solvent. Subsequently, the obtained mixture was purified via a column chromatography on silica and a viscous yellow liquid was obtained.

CNTs were first treated by sulfuric acid and nitric acid to obtain CNTs-COOH as reported in the literature. The CNTs-COOH $(0.15 \mathrm{~g})$ was dispersed in DMF $(50 \mathrm{~mL})$ with an ultrasonic homogenizer. Then the acquired CNTs-COOH suspension, $\mathrm{Et}_{3} \mathrm{~N}$ $(0.15 \mathrm{~g}, 1.50 \mathrm{mmol})$ and CTA $(0.15 \mathrm{~g}, 0.30 \mathrm{mmol})$ were added to a two-necked flask with stirring for $24 \mathrm{~h}$ at $60{ }^{\circ} \mathrm{C}$. The obtained products were washed with water and anhydrous ethanol for three times in turn and then dried in a $50{ }^{\circ} \mathrm{C}$ under vacuum. 
<smiles>CCO[Si](CCCNC(=O)CCNCCC[Si](OCC)(OCC)OCCCNC(=O)C(C)(C)Br)(OCC)OCC</smiles>

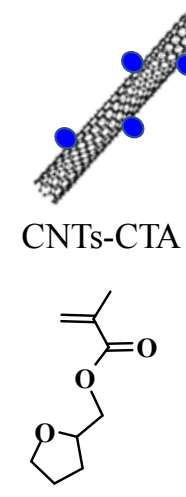

TMA

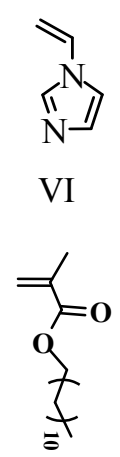

LA

\section{RAFT}

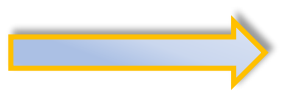

Figure S1. Schematic illustration for synthesis of CTA and CNTs-g-P(TMA-co-LAco-VI)/Zn (CHSMPs). 


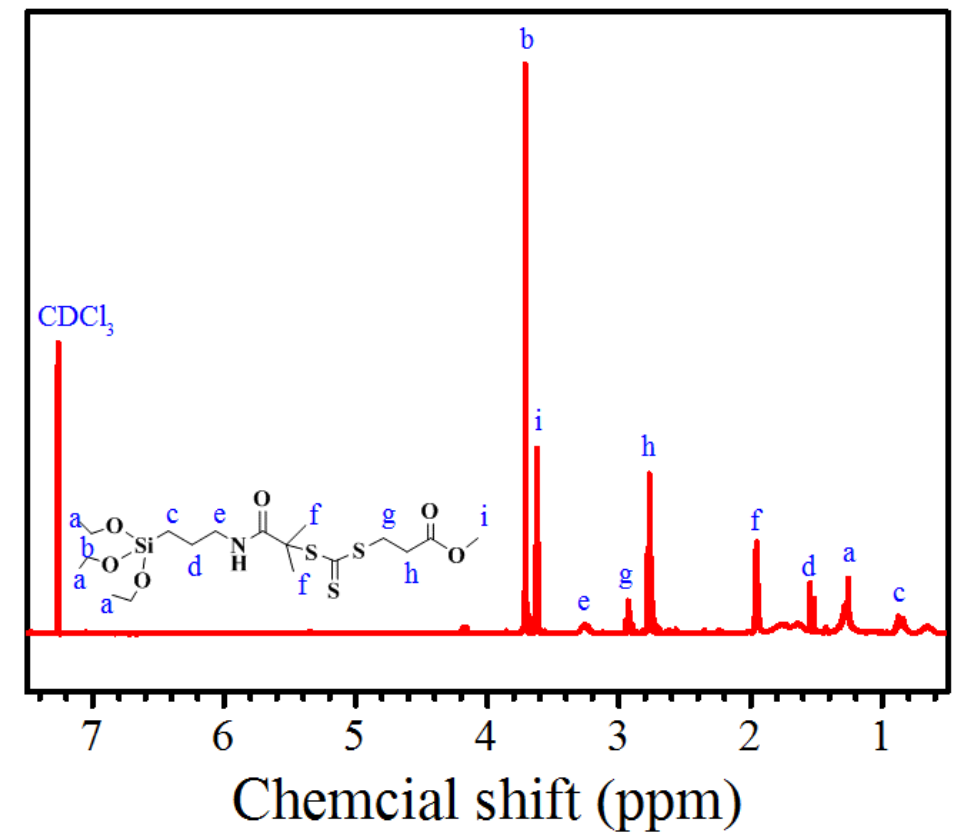

Figure S2. ${ }^{1} \mathrm{H}$ NMR spectrum for for CTA.

$\left(\mathrm{CDCl}_{3}, 500 \mathrm{MHz}\right) . \delta 3.71 \mathrm{ppm}(\mathrm{q}, 6 \mathrm{H}), 3.62 \mathrm{ppm}(\mathrm{t}, 3 \mathrm{H}), 3.26 \mathrm{ppm}(\mathrm{m}, 2 \mathrm{H}), 2.92 \mathrm{ppm}$ (t, 2H), 2.77 ppm (t, 2H), 1.96 ppm (s, 6H), 1.55 ppm (s, 2H), 1.25 ppm (m, 9H), 0.88 $\operatorname{ppm}(\mathrm{q}, 2 \mathrm{H})$. 

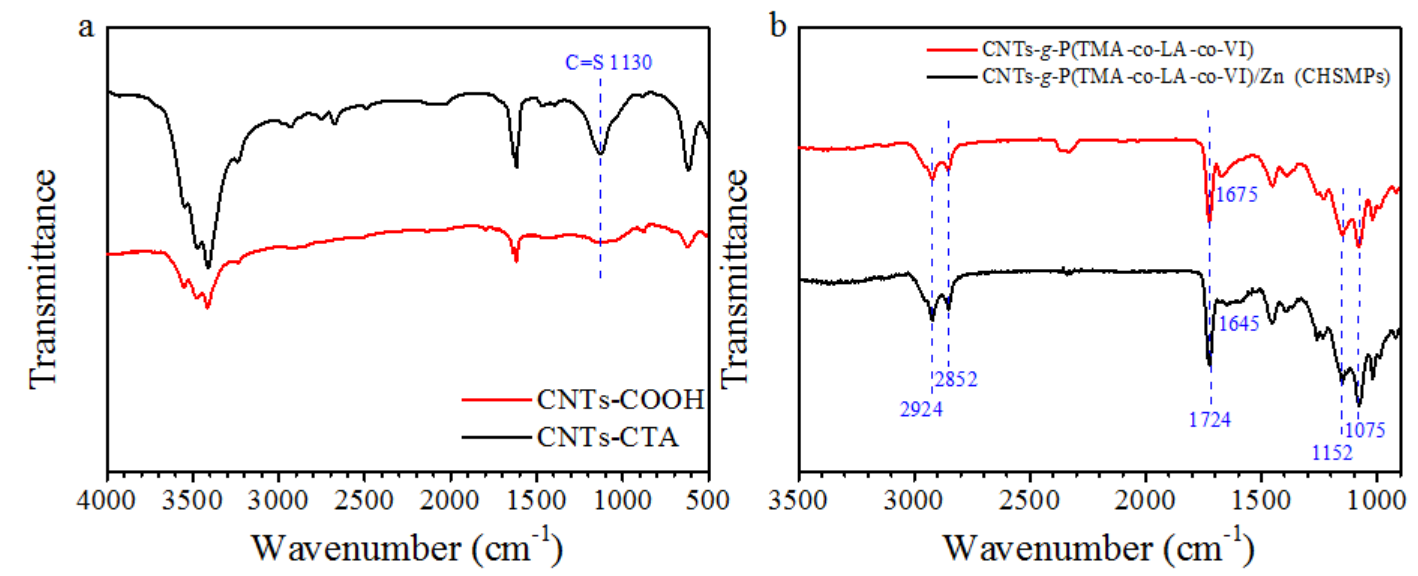

Figure S3. (a) FT-IR spectra of CNTs-COOH and CNTs-CTA. (The absorption bands at $1130 \mathrm{~cm}^{-1}$ for the $\mathrm{C}=\mathrm{S}$ bond in CNTs-CTA can be clearly seen, indicating the successful synthesis of CNTs-CTA.) (b) FT-IR spectra CNTs-g-P(TMA-co-LA-co-VI) and CNTs-g-P(TMA-co-LA-co-VI)/Zn (CHSMPs). (The stretching vibration absorption peaks of $\mathrm{CH}_{2}$ in LA side chain appear in $2924 \mathrm{~cm}^{-1}$ and $2852 \mathrm{~cm}^{-1}$. And the sharp strong absorption peak at $1724 \mathrm{~cm}^{-1}$ was assigned to the stretching vibrational absorption of the $\mathrm{C}=\mathrm{O}$ bond. The characteristic absorption peaks appear at $1152 \mathrm{~cm}^{-1}$ and $1075 \mathrm{~cm}^{-1}$ are generated by $\mathrm{C}-\mathrm{O}-\mathrm{C}$ stretching vibration. The absorption peak of $\mathrm{C}=\mathrm{N}$ shifted from $1675 \mathrm{~cm}^{-1}$ to $1645 \mathrm{~cm}^{-1}$ after crosslinking, indicating a successful crosslinking process.) 


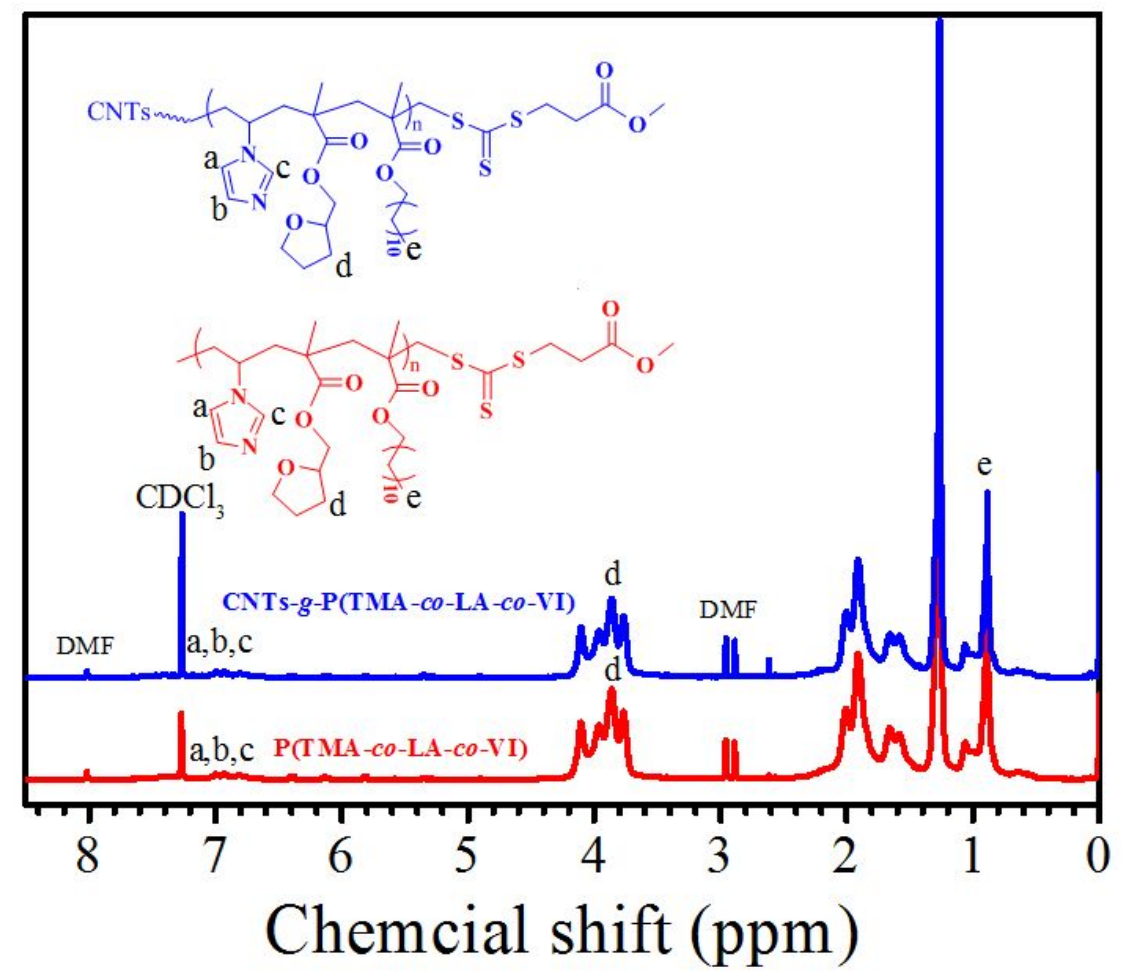

Figure S4. ${ }^{1} \mathrm{H}$ NMR spectrum for CNTs- $g$-P(TMA-co-LA-co-VI) and P(TMA-co-LA$c o-\mathrm{VI})\left(\mathrm{CDCl}_{3}, 500 \mathrm{MHz}\right)$.
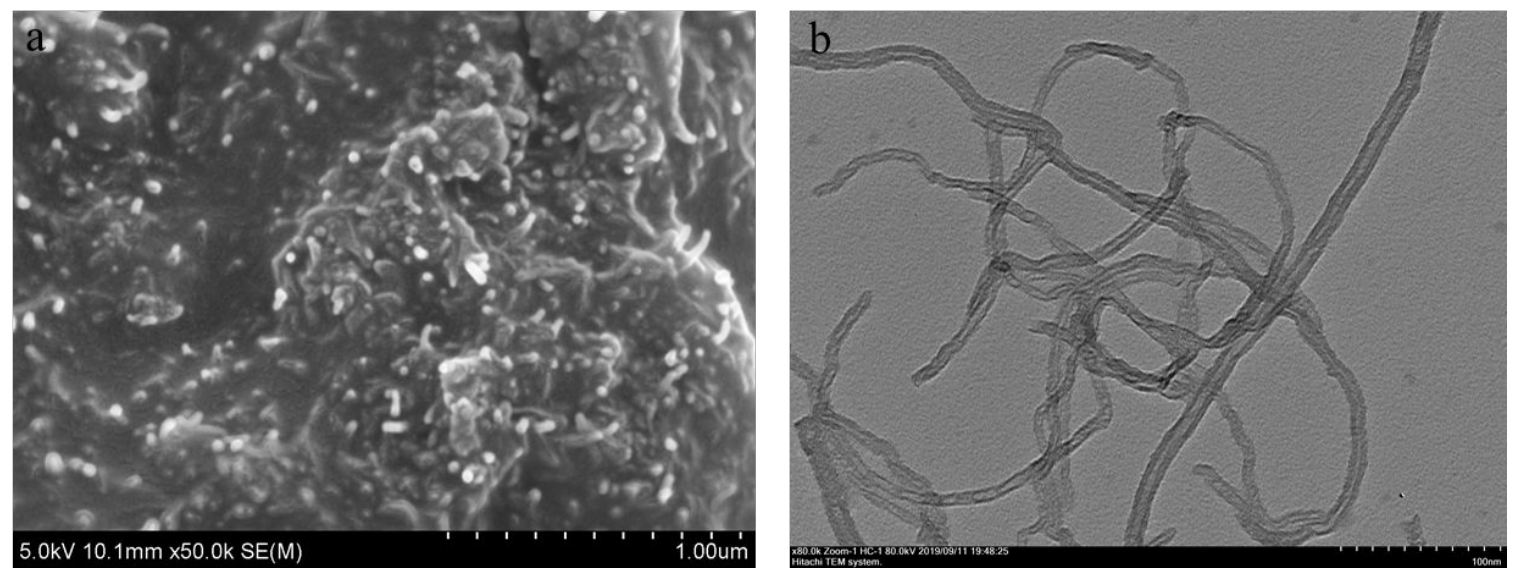

Figure S5. (a) SEM images of the cross-sectional view of CHSMPs film. (b) TEM images of the CHSMPs film. 


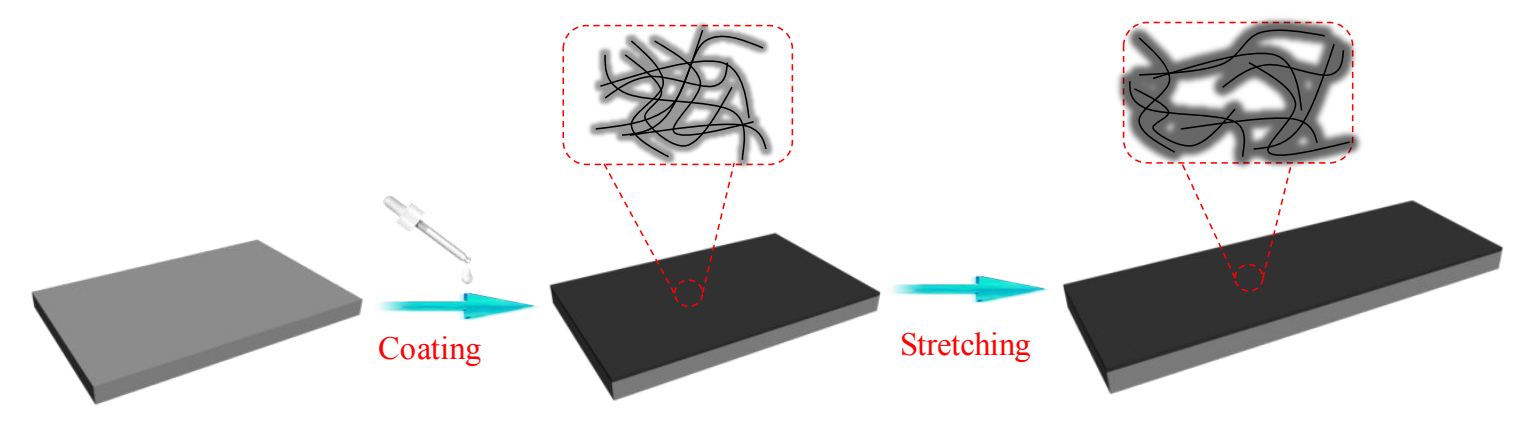

Figure S6. Schematic illustration for the synthesis of CNTs/CNTs- $g$-P(TMA-co-LAco-VI)/Zn (CNTs/CHSMPs).
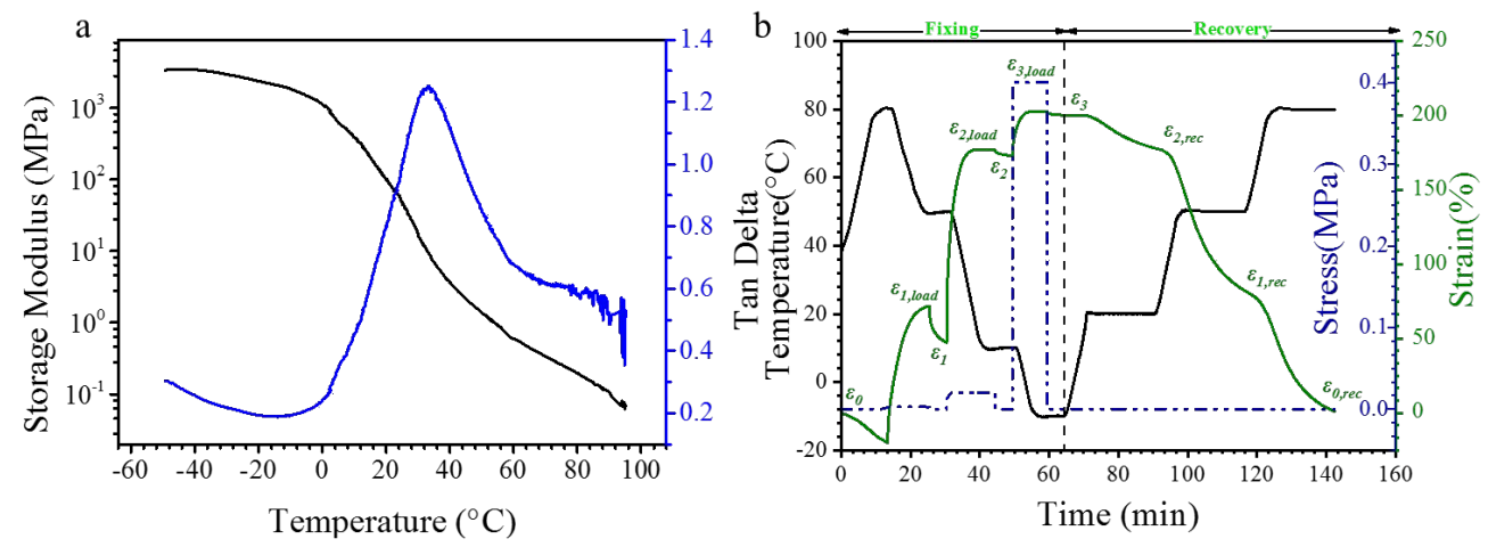

Figure S7. (a) The changes of storage modulus and $\tan \delta$ versus temperature for CHSMPs sample. (b) Quadruple-shape memory cycle of the CHSMPs sample.

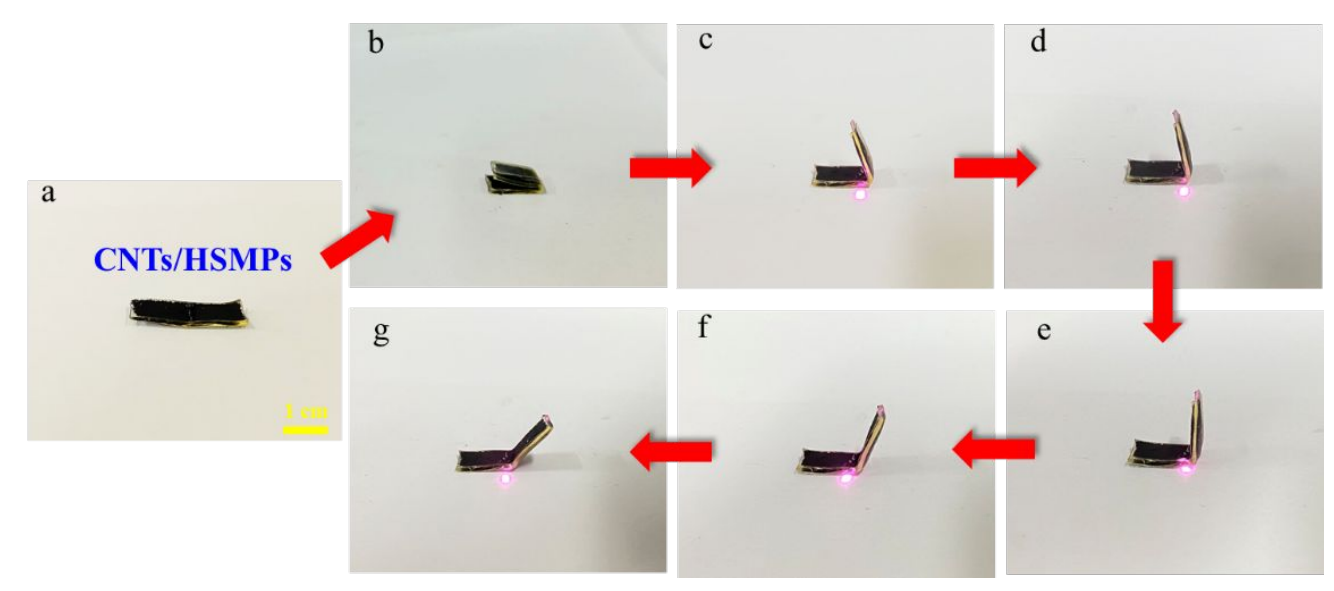


Figure S8. (a-g) NIR light-actuated shape recovery process of CNTs/HSMPs sample.
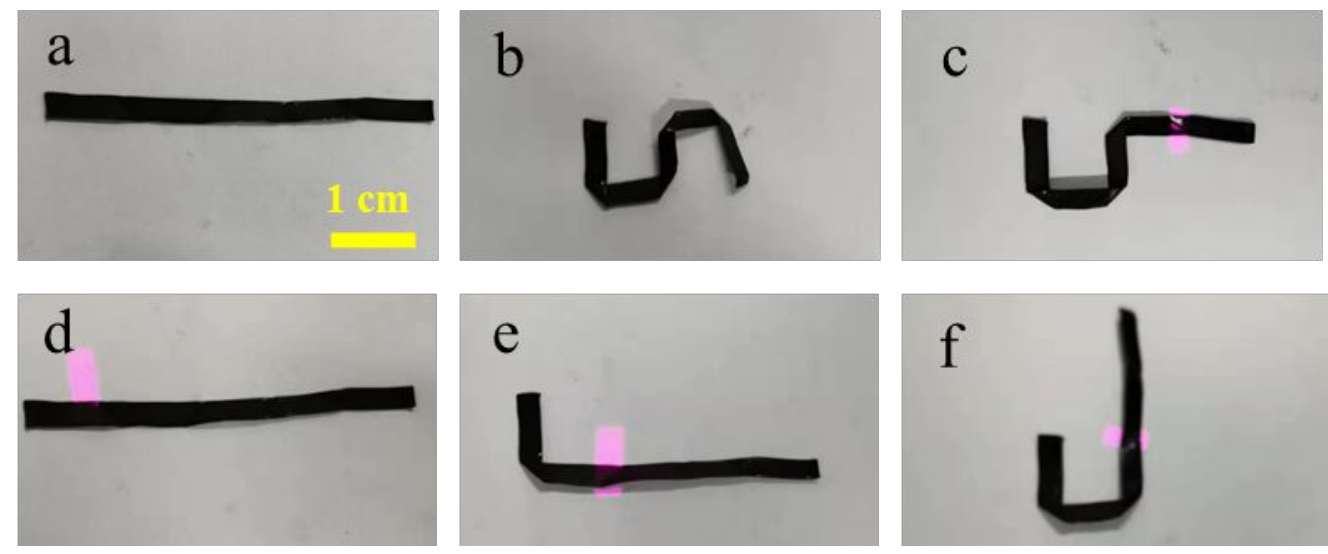

Figure S9. (a-f) NIR light-actuated targeted shape-morphing process of CNTs/CHSMPs sample.
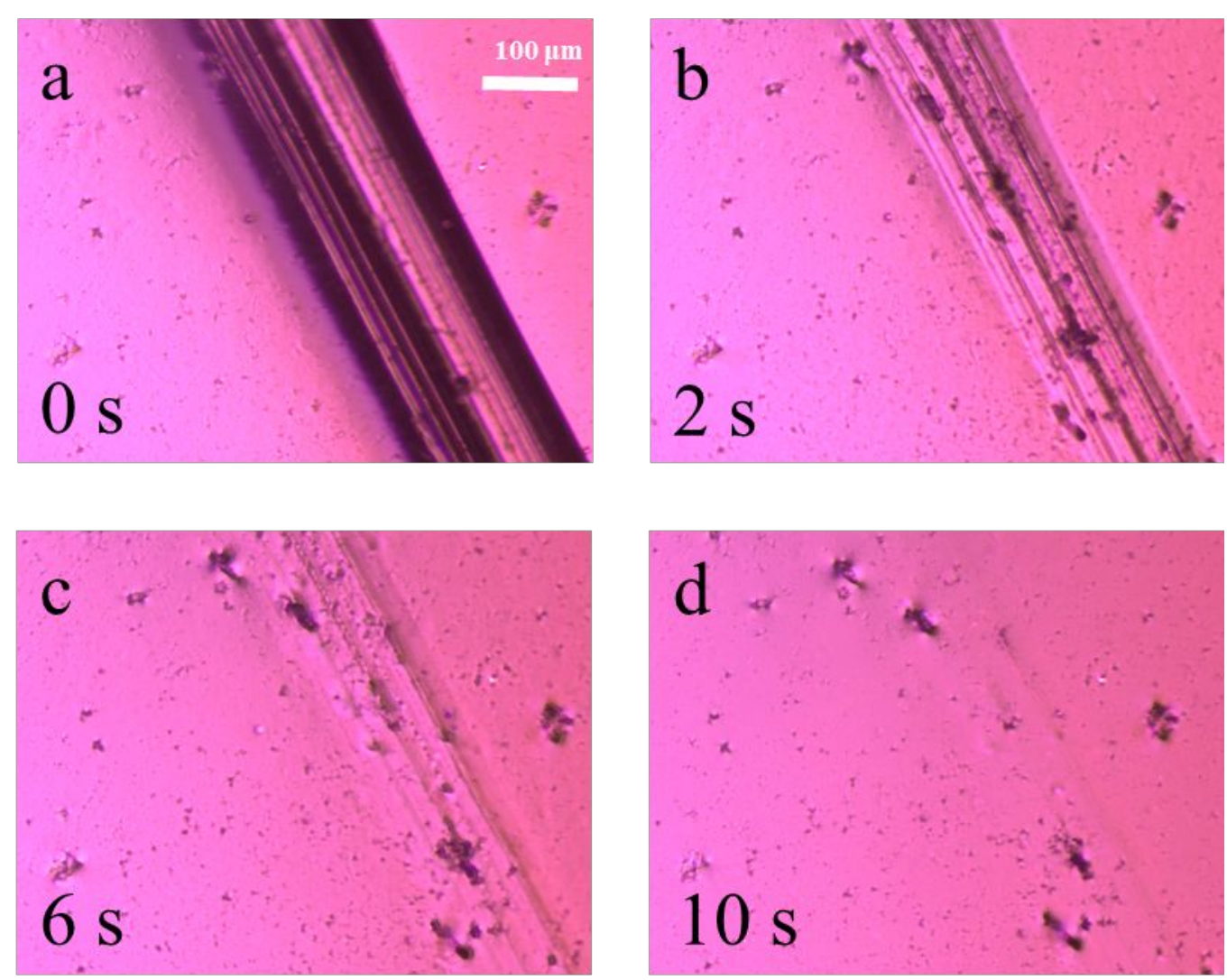

Figure S10. (a-d) NIR light-actuated self-healing process of the CHSMPs sample was 
observed by a polarizing microscope. 


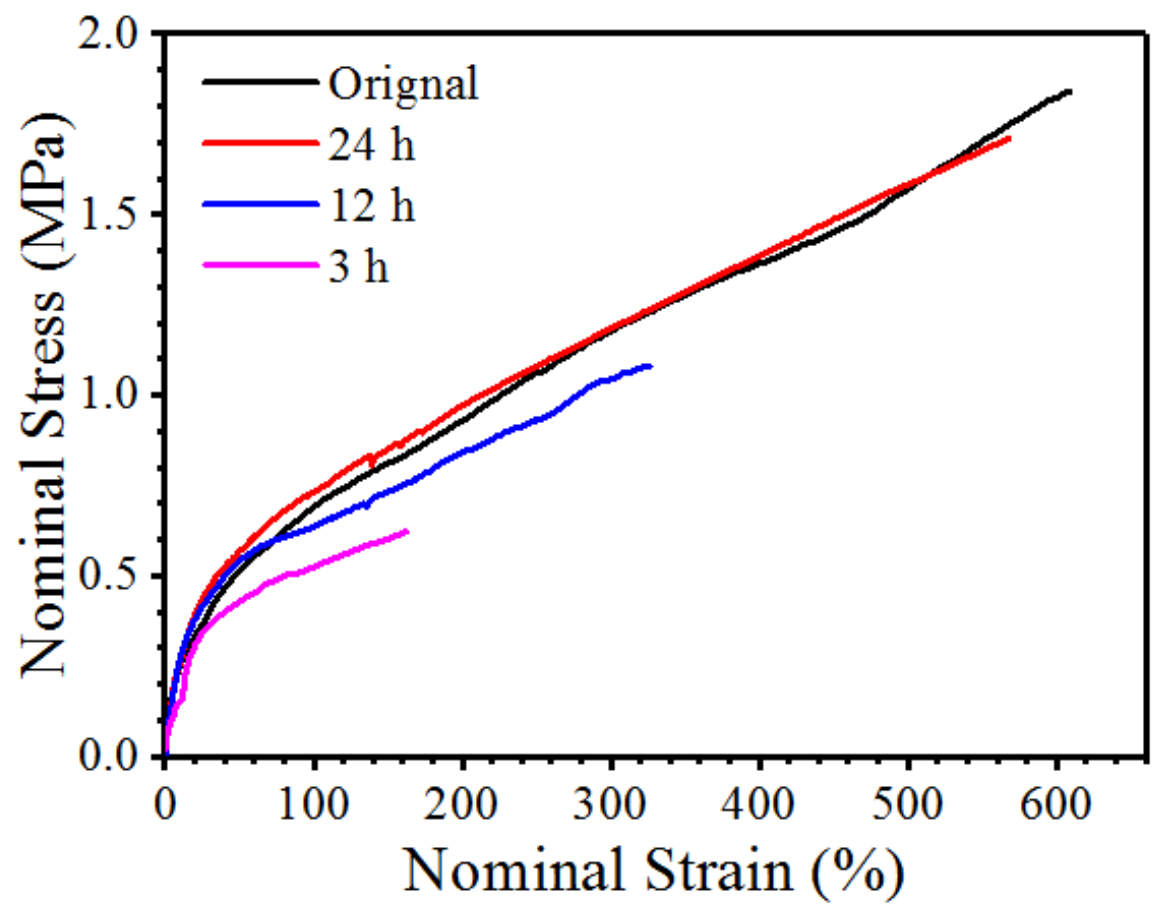

Figure S11. Typical tensile stress-strain curves of original and healed specimens using heating at $70{ }^{\circ} \mathrm{C}$ 